\title{
Expandiendo la noción de profesionalidad docente desde la educación geográfica'
}

\author{
Victor Salinas Silva² y Clare Brooks ${ }^{3}$
}

\begin{abstract}
RESUMEN
En este artículo teórico se discuten las conceptualizaciones sobre práctica docente en geografía. Se toma posición desde la didáctica específica de la geografía argumentando que su desarrollo teórico provee referentes de profesionalidad a los profesores para agenciar las problemáticas del contexto educativo tomando como punto de apoyo su "experticia" en geografía. Se revisaron 75 publicaciones en educación geográfica seleccionadas de acuerdo a la membresía de sus autores al Comité de Educación Geográfica de UGI, Red Latinoamericana de Didáctica de la Geografía y Sociedad Chilena de Ciencias Geográficas. La discusión identifica una brecha entre los estudios sobre didáctica de la geografía en Iberoamérica y curriculum making del contexto anglosajón; y, otra entre la conceptualización de práctica docente y práctica profesional. Por último, se teoriza sobre la necesidad de expandir la noción de práctica transitando hacia la de "experticia" a de los profesores, ya que considera su proceso de especialización en los contextos.
\end{abstract}

Palabras clave: Experticia, práctica docente, didáctica, educación geográfica.

\begin{abstract}
The objective of this conceptual paper was to explore how teachers' practice was problematised in the field of geography education. Research in geography didactics and curriculum making are two fields that have been relevant to build the understanding on teachers practice in geography. The challenge for geography education therefore appears to be how to understand the relationships between the two in coherent and systematic ways. This paper engages with the specific meaning of being a subject specialist and the gap in the literature between curriculum making and didactics to understand that specialisation. A second gap is identified regarding the relationship between teacher practice and the professional practices encircling in-classroom activities. The paper finalises suggesting the use of the term expertise to address the dynamics of specialisation in a subject but also with its implication to teachers' professional practice.
\end{abstract}

Keywords: Expertise, teacher practice, didactics, curriculum making, geography education. 
Diferentes comunidades de educadores de geografía en el mundo se han articulado para discutir y redefinir lo que significa la práctica docente en geografía. En Inglaterra esto se ha plasmado en el manifiesto "A Different view" de la Geographical Association (GA, 2009), en Estados Unidos se desarrolló el "Road Map for 21st Century Geography Education" de la Association of American Geographers (Bednarz et al., 2013) o "Una educación geográfica para Chile" (Arenas et al., 2016) de la Sociedad Chilena de Ciencias Geográficas. En estos documentos se habla de un momento de cambio para la profesión docente e incertidumbre sobre la supervivencia de las asignaturas, que requiere discusiones complejas sobre capacidades, infraestructura y concepciones que dan soporte a las prácticas de la geografía escolar.

El problema de definir la práctica docente, es que involucra considerar las nociones sobre profesionalidad que describen lo que constituye ser un "buen profesor". Existen diferentes marcos teóricos que reivindican para sí esta cualidad. Los marcos del profesor efectivo (Barber \& Mourshed, 2007), competencias (Blay, 2013; Pages, 2007), curriculum making (Brooks, 2013; Catling, 2013; Lambert \& Morgan, 2010) y didáctica (Araya, 2005; Arenas, 2009; Souto, 2013; Souza Cavalcanti, 2012) tienen objetivos similares pero con sutiles diferencias en su conocimiento de base. Por ello, cada marco teórico se encuentra influenciado en distinta forma por las diversas tendencias en educación que hiper-socializan (Mitchell, 2016) o politizan (Morgan, 2009) las prácticas docentes. En este artículo se toma posición desde la didáctica específica de la geografía argumentando que su desarrollo teórico ha permitido proveer referentes de profesionalidad a los profesores para agenciar las problemáticas del contexto educativo tomando como punto de apoyo su "experticia" en geografía.

Concentrarse en la práctica de los profesores, puede involucrar múltiples perspectivas curriculares o de evaluación, pero un enfoque en didáctica permite generar comprensión sobre lo que significa entrar a las aulas y los contextos que las influencian. Sobre esto Roberts (1995) sugiere que cuando los profesores se enfrentan a nuevas recomendaciones curriculares, hay un momento de apropiación de los nuevos requerimientos, pero que en breve los profesores tienden a volver a las prácticas y concepciones que ya han probado y experimentado. Para Brooks (2013: 83) la relación entre práctica y los cambios estructurales del sistema escolar no son necesariamente directos, la efectividad en la relación puede estar condicionada por "el grado en que los profesores se relacionan con los cambios, y la medida en que los cambios están en sintonía con el contexto local y las influencias dominantes del contexto". Para cualquiera de los escenarios, se desprende que el grado en que se relacionan los profesores con la política o el curriculum depende del criterio profesional que aplican en su práctica, siendo fuente de autonomía e identidad profesional.

En el artículo se examinan las conceptualizaciones sobre práctica docente comenzando con una primera sección que describe la vigencia de las teorías de la escuela-efectiva. En la siguiente sección se introducen las nociones sobre práctica docente en geografía que han sido relevadas por la investigación en Iberoamérica (didáctica de la geografía) y el mundo anglosajón (curriculum making). Para esta última se ha mantenido el término en inglés ya que su traducción directa como "curriculum realizado" no capta su desarrollo como campo de estudios. En esta sección se relacionan ambos campos que provienen de tradiciones diferentes pero que cuentan con preocupaciones comunes respecto a la enseñanza de la disciplina. Se profundiza en la definición de la didáctica como un término equivalente al de práctica docente. La tercera sección mueve 
la discusión a los desafíos que afronta la práctica docente desde la perspectiva profesional. Por último, se teoriza sobre la necesidad de expandir la noción de práctica hacia la de "experticia" de los profesores en geografía.

\section{¿Qué (cómo) sabemos sobre las prácticas docentes?}

Existen dos convenciones generales que han informado el entendimiento sobre la práctica de los profesores en el aula. La primera, considera que la calidad de la práctica docente es relevante para el sistema escolar. En 2007 el informe McKinsey argumentó que los profesores pueden tener una incidencia relevante en los resultados de los estudiantes (Barber \& Mourshed, 2007). El reporte fue construido considerando investigación previa sobre el "efecto-escuela" donde los estudiantes en establecimientos con profesores experimentados aventajaban estudiantes de otras escuelas con similares características socio-económicas pero diferente calidad de profesores. La incidencia de este informe fue tal, que diferentes gobiernos en América Latina implementaron reformas educacionales enfatizando el rol de los profesores y siguiendo sus recomendaciones en materia de formación inicial y desempeño en el sistema (Darling, 2011).

La segunda convención que se maneja a nivel del sistema escolar, es que la calidad de la práctica docente ha sido definida utilizando como criterio el marco de la escuela efectiva. La que ha influenciado incluso la idea de profesionalidad que se tiene de los profesores (Ball et al., 2012; Mitchell \& Lambert, 2015). Ser un profesor efectivo involucra manejar el contenido, usar una gama de pedagogías y tener altas expectativas para motivar e involucrar a todos los estudiantes.

En Iberoamérica, el entendimiento sobre enseñanza efectiva ha estado vinculado con el modelo por competencias como una forma de proveer entendimiento sobre lo que se espera tener como productos finales de la educación (Pages, 2007). En esta línea, en 1997 los países miembros de la OCDE lanzaron el Programa Internacional de Evaluación de los Alumnos (PISA), a lo que le siguió el proyecto DeSeCo para la Definición y Selección de Competencias (OECD, 2005), proporcionando un marco para los nuevos dominios de competencia. Blay (2013) sugiere que la idea de una "persona competente" toma en consideración años de discusión de las teorías constructivistas sobre el aprendizaje. Resumiendo, sus elementos principales en un conjunto de dominios específicos. Indicando que este marco opera como un consenso que provee directrices sobre como comprender el aprendizaje y, por lo tanto, delimitando el rol de los profesores en el aula. De acuerdo a Vásquez (2016) los profesores deberían prestar atención a esta forma de comprender el aprendizaje considerado como conocimientos, habilidades y valores comunes. Siendo la combinación de estos tres una competencia. Desde una perspectiva teórica, este marco ha sido fructífero generando modelos educativos (Cisterna, 1999; Eggen \& Kauchak, 2005) que proveen pautas para enseñar distintas competencias. Por ejemplo, la enseñanza de habilidades para el pensamiento crítico, resolución de problemas o toma de decisiones han sido modelos que emergieron en este contexto. Modelos fuertes en lo pedagógico, con un componente curricular orientador que se sostiene en los avances en materia de evaluación.

Las distintas teorías sobre didáctica de la geografía han transitado en paralelo a las de la escuela efectiva, posicionándose como un underdog en el escenario educativo actual. Las lleva las 
de perder porque la geografía sigue siendo central en la didáctica de la disciplina. Suena contradictorio, pero es lo que pasa cuando se confunde conocimiento con contenido.

El estatus que goza el modelo por competencias también puede ser comprendido como un discurso que ha demarcado las formas de comprender las prácticas docentes a nivel de los sistemas escolares (Seow, 2016). Aunque las teorías relacionadas con el movimiento de la escuela efectiva han sido necesarias para acentuar la importancia de los docentes y sus prácticas de aula, su aplicación en programas de formación y requerimientos de política pública deja poco espacio para que los profesores puedan maniobrar e interpretar lo que están enseñando.

\section{La práctica docente en educación geográfica}

En respuesta a lo anterior -y como convención general en realidad, esto no representa un fenómeno novel- la literatura en educación geográfica subraya que la geografía no solo es un contenido, sino que ayuda a leer el contexto del profesor y dar voz a los territorios a través de la identificación de conocimientos locales. En este marco, la didáctica de la geografía resalta la "experticia" docente para manejar la interacción de distintos cuerpos de conocimiento y los actores que los manifiestan como válidos en sus propios espacios.

En este sentido, la segunda sección del análisis explora como la práctica de los docentes ha sido problematizada en el campo de la educación geográfica. Tanto la didáctica de la geografía en Iberoamérica y curriculum making en contextos anglosajones han sido dos campos de investigación que han contribuido al entendimiento sobre las prácticas de los profesores en geografía. Por lo tanto, el desafío que emerge para la educación geográfica es tratar de comprender la relación entre los dos campos de forma coherente y sistemática.

La didáctica -como campo de estudios- ha sido uno de los ejes impulsores de la educación geográfica en Chile (Araya, 2005; Arenas, 2009; Garrido, 2005) e Iberoamérica (Callai, 2016; Moreno Lache et al., 2013; Souto, 2013; Souza Cavalcanti, 2012; Vanzella Castellar, 2011). La didáctica considera la pedagogía involucrada en el acto de enseñar y, como campo de conocimiento busca caracterizar los elementos procedimentales requeridos para enseñar y aprender una materia en particular. Por ello, juega un rol de interacción al estar situada entre la pedagogía y el conocimiento, haciendo énfasis en cómo el aprendizaje es generado en determinados contextos educacionales. Otras dimensiones han sido añadidas de forma circunstancial pero las dos dimensiones anteriormente señaladas son claves para la comprensión de una didáctica específica de la disciplina.

La noción de didáctica ha cambiado con el tiempo. Originalmente ha sido asociada con los métodos, técnicas y recursos que los profesores usan para impartir sus clases (González, 2002). Usar guías de trabajo, imágenes u otros recursos en una clase han sido -y todavía lo son- considerados como algo "didáctico". El término se ha mantenido porque retiene su significado original haciendo referencia a lo que se entiende por "buena enseñanza".

El tránsito de la didáctica, desde estar centrada en los recursos y el profesor hacia estar centrada en el aprendizaje, se institucionalizó en la década de 1990, considerando especialmente cómo los estudiantes aprenden y aprenderían una materia específica (Pages, 2007). Fue el sello 
del constructivismo y reflejo de las nuevas demandas sobre el sistema educativo (Coll, 1993; Coll et al., 1992).

Tal preocupación por los estudiantes no es nada nuevo. Vergara y Cofré (2014: 327) sugieren que esta preocupación se puede rastrear a la tradición de la didáctica específica que se encuentra arraigada en la educación chilena. Debido a la influencia europea en la línea española y francesa, pero también con una raigambre en la tradición alemana que "posee la "Fachdidactik" o didácticas de los contenidos disciplinares, la cual se diferencia de la didáctica general (...) en su característica de disciplina dedicada a estudiar las estrategias específicas de enseñanza de un contenido". De este modo, se puede entender que la didáctica se actualiza en el escenario constructivista, pero antecede a modelos como los de Shulman et al. (1987) relacionados con el Conocimiento Pedagógico del Contenido (PCK -por sus siglas en inglés-) y que fueron popularizados por el influjo de los estudios cognitivas en educación. En vez de re-organizar una forma completamente nueva de relacionar pedagogía y conocimiento (Arenas \& Salinas Silva, 2013), los nuevos modelos cognitivistas simplemente se acomodan a una estructura previa de enseñanza (Chaparro, 1967) que la tradición en didáctica ya había producido.

La forma en que se quiere producir aprendizajes también ha visto en la didáctica una preocupación por la generación de aprendizaje profundo y conceptual en los estudiantes. De acuerdo a Araya (2005) el foco epistémico de la disciplina contribuye a generar comprensión sobre cómo la geografía se ha construido. Permitiendo que en la práctica docente se pueda reflexionar sobre cómo los estudiantes pueden pensar geográficamente y, por lo tanto, haciendo más manejable el contenido geográfico (Souto, 2013; Souza Cavalcanti, 2012; Vanzella Castellar, 2011).

La didáctica específica de una disciplina se encuentra organizada de forma diferente a otros campos pedagógicos en educación debido a que enfatiza la especificidad de aprender un set particular de conocimientos. Si se utilizan las publicaciones científicas como referente, se podrá notar que el mayor grupo de artículos en el campo de la didáctica de la geografía en Chile son publicados en revistas de geografía. La Revista de Geografía Norte Grande (Araya, 2005; Arenas \& Salinas Silva, 2013; Errázuriz et al., 1997) Revista Geográfica de Valparaíso (Chaparro, 1967; Dentice y Garrido, 2013), Revista Espacios (Gallastegui, 2015; Salgado, 2012) o Scripta Nova (Araya Palacios et al., 2015). Por lo general este tipo de publicaciones comparten referencias comunes e incluso presentan casos de citación cruzada entre autores. Otro grupo de artículos se encuentran publicados en revistas de educación como es el caso de Estudios Pedagógicos (Reyes et al., 2013), Diálogos Educativos (Llancavil \& González, 2014) o Íber (Cisterna, 1999), que son trabajos que tienen marcos conceptuales diversos dependiendo de los problemas educacionales a los que se están refiriendo. Separado de estos dos grandes grupos existen otras contribuciones que vienen desde geógrafos investigando problemáticas geográficas en el ámbito educativo (Paulsen et al., 2012; Sabani \& Moreira, 2014). La organización de estos tres grupos de publicaciones sugiere que las "culturas de influencia" (Brooks, 2007) representadas en el campo de la didáctica se encuentran construidas tanto desde la cultura investigativa de la Geografía, como de la Educación, pero de forma separada.

Esto complejiza el argumento de Stengel (1997), quien sugiere que las asignaturas escolares se encuentran en cierta medida conectadas con su par académico. Para Young (2011), esta co- 
nexión permite que ese conocimiento en específico sea confiable, falible y se pueda testear. La posibilidad de que el conocimiento geográfico en el contexto educativo se pueda rastrear hasta sus fuentes y referentes significa que las prácticas docentes desde la perspectiva didáctica tienen trazabilidad y pueden responder a una examinación de su validez.

Quizás la problemática Stengel-Young puede ser resuelta considerando algunos de los postulados desarrollados en el marco del campo de curriculum making. Para la Geographical Association, curriculum making puede ser entendido como el balance entre "tres fuentes de energía en la sala de clases: la "experticia" y destrezas prácticas del profesor; el interés y necesidades de los estudiantes, lo que la disciplina dinámica y cambiante puede ofrecer" (Geographical Association, 2009, p. 27). Esta definición se encuentra expresada en su manifiesto a propósito de la renovación del currículo escolar en Inglaterra. Se encuentra en cierta medida alineado con las concepciones que Marsden (1997) ha expresado sobre el perfil del profesor especialista en su materia. Indicando que la práctica docente debe encontrar un balance entre el contenido de la asignatura, los procesos educacionales y el propósito social de estos. Tal posición sugiere que el campo de curriculum making asume un rol que se enfoca en la interacción entre estas dimensiones y es única a la experiencia del profesor especialista. De acuerdo a Lambert \& Morgan (2010), esto significa que el foco de este campo de estudios no se encuentra solo en la planificación o diseño curricular, sino más bien en las acciones y elementos técnicos que los profesores resuelven en el día a día.

Tanto curriculum making como la didáctica, comparten la diferenciación entre la existencia de un curriculum prescrito nacional y un curriculum realizado en el aula (Catling, 2013; Souto, 2013). Ambos se centran en la importancia de la profesionalidad docente y la posición autoritativa del profesor para definir su práctica pedagógica (Arenas \& Salinas Silva, 2013; Brooks, 2013). Por lo que buscan contribuir en la profesionalidad y el conocimiento de base requerido en la toma de decisiones de los profesores. Aunque los campos de curriculum making y didáctica no son lo mismo, comparten preocupaciones comunes.

Una de las principales diferencias entre las dos líneas de estudio es su relación con el curriculum. La base del entendimiento sobre curriculum making se encuentra en los estudios sobre el curriculum realizado (Lambert \& Morgan, 2010) indicando un foco sobre las prácticas de los profesores en el aula. De acuerdo a Catling (2013: 443) "para la GA el termino se focaliza en la planificación de mediano-plazo, derivada de un esquema de trabajo que se convierte en la base de lo que se realiza en la clase". No obstante, para Huber et al. (2011) y Dentice y Garrido (2013) el problema con la literatura sobre curriculum realizado es que inevitablemente compara la práctica de los profesores con el curriculum prescrito nacional. Sugiriendo que la validez de lo que se enseña y como se enseña proviene de fuentes externas a la práctica misma de los profesores. Al contrario, la didáctica del profesor se posiciona como un elemento autónomo respecto de las especificaciones del curriculum. Araya (2005) considera que la didáctica no es solo el acto de interpretar los objetivos curriculares, sino que se concibe como ambos: una expresión de la práctica docente y; una forma de conocimiento para comprender los límites de la profesión docente.

Una diferencia subyacente a ambas líneas de trabajo tiene que ver con la forma de entender los diferentes componentes de la docencia. Un caso en específico es la conceptualización que se tiene sobre las habilidades. Estas han sido relevadas para que los estudiantes puedan participar activamente en la sociedad (Esteves, 2012). No obstante, existen diferencias en las líneas de tra- 
bajo iberoamericanas y anglosajonas. En España (Coll, 1993; Monereo, 1997) se ha enfatizado el rol de las habilidades cognitivas para comprender el aprendizaje como un proceso psicológico. Roberts (2013) sugiere la importancia que ha tenido en esta línea el uso de taxonomías que delinean un orden jerárquico de habilidades, como es el caso de la taxonomía de Bloom que sitúa por ejemplo el "analizar" como una habilidad de orden superior respecto del de la localización. Por el contrario, la literatura anglosajona se concentra en las acciones necesarias para completar una tarea. Por ejemplo, para Catling \& Willy (2009) el conocimiento locacional es acerca de saber dónde se encuentran los lugares, pero el conocimiento sobre el "lugar" busca saber cómo son estos y cómo generan cierta familiaridad con los estudiantes (Scoffham, 2010).

La diferenciación en la forma de comprender las habilidades puede llegar a ser controversial. Para el profesor involucra discriminar qué particular set de habilidades debería desarrollar y con qué "experticia" tratarlos para apoyar el aprendizaje de los estudiantes, así como los diferentes conocimientos que tienen que ser operacionalizados para lograr tal objetivo. Sin embargo, ambas formas de comprender las habilidades han sido explicadas por Pozo y Postigo (2000) como conocimiento procedimental. Los procedimientos son reconocidos como contenido escolar que acompaña la comprensión sobre los conceptos y principios geográficos. Lana Cavalcanti (1998) e Isidoro González (2002) difieren en qué medida los procedimientos están en relación con los métodos y enfoques que una disciplina usa para construir conocimiento. No obstante, existe certeza que los procedimientos están directamente involucrados en el andamiaje requerido por los estudiantes para aprender a pensar geográficamente (Roberts, 2003). Este ejemplo permite ilustrar como los dos campos tienen diferencias estructurales relacionadas con sus tradiciones, pero que al mismo tiempo comparten concepciones subyacentes acerca de lo que implica desarrollar prácticas específicas y el dominio que requiere por parte de los profesores.

Un problema inherente a la práctica docente es el desarrollo de "experticia" respecto al conocimiento del contenido y la cuestiones que puede presentar su validez (Brooks \& Hopwood, 2005). En algunos trabajos sobre curriculum making y la agencia de los profesores, se visualizan tensiones sobre la definición del conocimiento que está informando el aprendizaje (Hart et al., 2014), donde se subraya la importancia de criticar el conocimiento del contenido y su rol en la práctica docente. Para Huber et al. (2011) existe una incompatibilidad entre el conocimiento disciplinar que requieren los contenidos escolares y otros tipos de conocimiento. Esta crítica se refuerza con los efectos que pueden causar las evaluaciones estandarizadas y la existencia de curriculums nacionales de carácter prescriptivo. No obstante, la incompatibilidad que este tipo de estudios reporta puede ser en cierta medida equivoca. Principalmente, porque esa crítica es una generalización y no considera que cada asignatura escolar tiene un diferente enfoque en la forma de comprender la realidad que está estudiando (Lambert, 2009). En el caso de la geografía, el mismo argumento esgrimido por Huber et al. (2011) acerca de la posibilidad de incluir conocimientos alternativos, ha sido levantado como un asunto relevante para el conocimiento del contenido geográfico.

Margaret Roberts (2003), Simon Catling (2014) y Lana Cavalcanti (1998) han argumentado en diferentes contextos sobre la importancia del conocimiento cotidiano para la enseñanza de la geografía. Roberts se refiere al problema partiendo desde una base constructivista que toma en consideración la particular forma de construir conocimiento en geografía a través de métodos investigativos. Su perspectiva considera que los constructos sobre el mundo no son fijos y, por lo 
tanto, modificados continuamente. En la práctica, esto significa que los profesores pueden tomar en consideración los conocimientos previos de los estudiantes para proveer oportunidades que les permitan reconstruir el conocimiento en dialogo con otros. Catling establece que la pedagogía asociada a la geografía puede empoderar a los niños revalorizando prácticas comunes en la geografía escolar como "el conocimiento de lugares en el mundo, la experiencia geográfica de los niños, sus respuestas a situaciones locales y globales y el aprendizaje desde la vida de niños en contextos de riesgo ambiental" (Catling, 2014: 352). Catling enfatiza las geografías personales y las nociones de identidad para comprender el contexto de los estudiantes como medio para involucrarlos en el aprendizaje. La posición de Cavalcanti es igualmente positiva respecto del conocimiento cotidiano y el conocimiento sobre los lugares, pero su enfoque previene respecto al uso de estos elementos simplemente como una estrategia para llamar la atención de los estudiantes utilizando sus experiencias personales. La investigación de estos tres autores permite ilustrar la necesidad de comprender que la "experticia" de los profesores sobre el contenido es un proceso de especialización sobre el conocimiento geográfico que les permite moverse desde el contexto de los estudiantes hacia el de la escuela y viceversa. De este modo, el perfil de un profesor especialista permite cerrar la brecha señalada por Huber et al. (2011) para el caso de la geografía escolar.

La discusión anterior enfatiza la importancia del conocimiento práctico y personal de los profesores, que de acuerdo a Huber y Clandinin (2011) y Dentice y Garrido (2013) puede estar en conflicto con la posicionalidad de la práctica docente como parte de un espacio de educación formal. El conocimiento práctico es descrito como el "cuerpo de convicciones y significados, conscientes o inconscientes, que han emanado de la experiencia (intima, social y tradicional) y que son expresadas en las prácticas de una persona" (Huber et al., 2011: 3). Para los autores, se requiere tomar distancia de las nociones tradicionales sobre práctica docente ocurriendo en las escuelas y hacer visibles otros espacios y personas que puedan involucrarse en la experiencia curricular de los niños y jóvenes. De forma similar, Dentice y Garrido (2013, p. 63) argumentan que la escuela es "estática y rígida en relación con su medio socio-espacial". Su crítica proviene de investigación en espacios indígenas donde se plantea que la escuela -como institución social- ha descuidado la experiencia de los estudiantes a través de homogeneización y discursos de identidad nacional. La crítica de Huber et al. (2011) es correcta pero apuntando de forma general a la "escuela", simplificando el estatus profesional de los profesores como miembros constitutivos de esa institución. Es correcto que los profesores tienen que seguir requerimientos nacionales, pero también tienen preparación y "experticia" que tiene que ser tomada en cuenta. Por otro lado, el argumento de Dentice y Garrido (2013) ha pasado por alto la problematización de la didáctica y curriculum making sobre el aula como un espacio de interacción. Como espacio en disputa (Seow, 2016) las practicas pedagógicas en geografía pueden ser vistas como una lectura cuidadosa del contexto en relación con espacios personales, profesionales, de barrio o comunitarios que van más allá del aula y son parte del ejercicio profesional de los docentes.

\section{La práctica profesional de los profesores: Expandiendo la definición de práctica docente}

Las prácticas de los profesores no son lo único que ocurre en la sala de clases. La práctica profesional hace referencia a las acciones que los profesores desarrollan fuera del aula para afirmar 
su propio trabajo o influenciar a otros para que los apoyen en su labor. Parte de la investigación en educación geográfica ha progresado en torno a esta área. Por ejemplo, Clare Brooks (2007) y Andoni Arenas (2009) han demostrado inquietudes similares referidas a las influencias que inciden en el profesionalismo de los profesores en relación con su "experticia" en geografía. En sus trabajos recientes Brooks ha enfatizado el papel de la identidad profesional docente (Brooks 2016). Mientras que Arenas se ha centrado en el desarrollo profesional de profesores con especial énfasis en las concepciones de los profesores respecto a la disciplina (Arenas et al., 2016). Los trabajos de David Lambert (Lambert, 2009; Mitchell \& Lambert, 2015; Solem et al., 2013) y Fabián Araya (Araya, 2005; 2009; Araya-Ramirez, Quiros-Arias, \& Ruiz-Hernandez, 2015) han contribuido a avanzar en esta área de la educación geográfica, proporcionando las bases y direccionando problemas respecto a las capacidades de los profesores en el contexto escolar y la comunidad en que se sitúan.

El foco en los elementos profesionales de la práctica docente ha sido tratado dependiendo de los diferentes espacios en que los profesores se desenvuelven. Clandinin \& Conelly (1995) desarrollaron el concepto de "Paisaje del Conocimiento Profesional" (Professional Knowledge Landscape) para centrar la atención en los dilemas intelectuales y morales que los profesores experimentan al navegar un paisaje profesional "lleno con personas, elementos y eventos diversos y en diferentes relaciones" (Huber et al., 2011: 4). Enfatizando como los profesores se mueven de un espacio a otro: dentro y fuera de la sala de clases.

La dimensión profesional asociada con las prácticas dentro del aula es teóricamente más convencional. Por ejemplo, Darling (2011) subraya la forma en que los profesores interactúan entre uno y otro como un factor clave para construir comunidades de práctica. "Cooperación y colaboración entre los profesores ha sido repetidamente señalada como un elemento importante de la práctica profesional y puede incluir el intercambio de material instruccional, desarrollo del currículo, reuniones para discutir el progreso de los estudiantes y actividades de aprendizaje colectivas" (OECD, 2013: 35). En efecto, la investigación en educación geográfica (Conway-Gomez \& Araya Palacios, 2011; Lambert \& Morgan, 2010; Scoffham, 2010) ha considerado la colaboración entre educadores de geografía como uno de los principales elementos para expandir la comprensión -generalmente local- que tanto estudiantes como profesores tienen de la geografía. Apoyando el desarrollo de estrategias y pedagogías para ello. Pero también ha identificado las problemáticas que involucra desplegar tal tipo de acciones. Puttick (2016) ha analizado la relación entre las historias individuales y colectivas de los profesores en distintos departamentos de geografía, sugiriendo que donde "la autonomía, identidad, estatus y juicio de los profesores ha sido minado a través de la centralización de la toma de decisiones" existe una menor autoeficacia por parte de los docentes en su práctica de aula. Puttick (2016) sugiere que el status de los profesores dentro de los establecimientos juega un rol importante definiendo concepciones sobre el conocimiento disciplinar, especialmente en la relación entre aquellos experimentados y los que se integran como parte de su primer año de carrera. Esto incluye los distintos tipos de fuentes de conocimiento que son influenciados por la comunidad de práctica para ser usados en la sala de clase. Arenas et al. (2016) plantea lo contradictorio que puede ser criticar los curriculum prescritos solo usando fuentes ministeriales de información.

Otra variable a considerar, es la frontera de las capacidades profesionales del docente. Fuera de la sala de clases existen una diversidad de fronteras profesionales que los profesores hallan 
difícil de cruzar y esas delimitaciones actúan como barreras para mover conocimiento y "experticia" en torno al sistema escolar (Ainscow, 2015). Algunas de estas fronteras se encuentran en las mismas escuelas debido a que los profesores se encuentran ligados a sus labores individuales dentro del aula. Incluso, en escuelas menos efectivas, los profesores no tienen conocimiento de lo que sus colegas se encuentran realizando dentro de la escuela (Avalos \& Assael, 2006).

Más allá de los límites de la escuela, se encuentran otros establecimientos y organizaciones pero esto representa otra frontera que restringe al profesorado para agenciarse con algún proyecto personal o institucional (Klocker, 2007). Para Alexandre (2016) identificar las limitantes a la capacidad de acción de los profesores de geografía puede ser tan simple como comprender la forma de implementar los objetivos curriculares. El autor plantea que intervenciones externas pre-formateadas, como los curriculum por competencias, afectan de manera tan profunda la dinámica en ciertos establecimientos escolares, que llegan a trasponer el auto-concepto profesional de los profesores, la cual es parte de una dinámica compleja de relaciones:

La identidad profesional de los profesores en geografía es concebida como una red construida en torno a elementos que pertenecen a sus representaciones de la profesión, una red que es activada cuando los profesores son confrontados con un contexto inter-relacional específico, que requiere de su identificación o diferenciación respecto de un grupo (Alexandre, 2016: 166)

De acuerdo a McNamara et al. (2014: 16) esta práctica de moverse entre espacios profesionales constituye un apoyo en red implícito y difícil de definir, lo que ha sido una de la razones que ha permitido la proliferación de distintas políticas que intervienen los establecimientos educacionales:

“[Es] parte de la razón por qué es tan fácil para políticos reducir el conocimiento de los profesores a solo conocimiento del contenido (adquirido en el pregrado) y habilidades básicas para ser "competente" en el aula, concibiendo de forma reducida el conocimiento profesional y la profesionalidad docente".

El ambiente de trabajo de los profesores se ha poblado con nociones sobre lo que es ser un "buen" o "efectivo" profesor. Lo que involucra el conocimiento del contenido geográfico pero que también ha limitado a los profesores a ser técnicos que entregan contenidos curriculares (Solem et al., 2013). Por ejemplo, en España existen diferentes publicaciones listando competencias que guían a los profesores en el cumplimiento de una tarea (Blay, 2013; Cisterna, 1999). Desde el desarrollo de pensamiento crítico hasta el uso de tecnologías. Aunque estos trabajos son útiles, Morgan (2009) señala que muchas de las teorías ofrecidas a los profesores están basadas en un "idealismo" que sugiere que los cambios en las prácticas docentes son mejor llevadas cuando los profesores cambian su propia forma de pensar:

"Asume, implícita o explícitamente, una postura atomizadora de las relaciones sociales. Propone un contexto en que los profesores pueden moverse de forma libre, autónoma y como agentes individuales, y describe la práctica docente en los términos de dicho contexto. Por lo tanto, el buen profesor tiene la capacidad para influenciar a los estudiantes como individuos trayendo transformaciones personales y educativas. Esto ignora la complejidad de las relaciones profesor-estudiante que son afectadas por relaciones de poder y control" (Morgan, 2009: 30). 
En la vereda opuesta, se posicionan teorías enfatizando la agencia y autonomía de los profesores como alternativas al actual paisaje profesional, que si bien tienen una visión positiva del profesor, forman parte del mismo problema. La idea sobre agencia docente debe ser tratada con cuidado, ya que puede ser engañosa al crear una falsa creencia donde las escuelas pueden cambiar sistemas sociales completos (Gleadle et al., 2008) o impedir la falta de poder de comunidades vulnerables (Danaher et al., 2014).

La premisa que estas teorías alternativas quieren disputar es difícil de contra-argumentar: profesores efectivos pueden mejorar los resultados de los estudiantes sin importar su condición socio-económica (Barber \& Mourshed, 2007). El modelo del profesor con altas capacidades técnicas (Coffield, 2012) acompañando la teoría de la escuela efectiva no se ha desarrollado solo porque es parte de las relaciones de poder de los sistemas escolares, sino porque es funcional y ha generado un determinado tipo de pedagogía. Ball (2012) ha argumentado en contra de este modelo señalando las condiciones de presión, competencia y rendición de cuentas que genera. No obstante, desde la educación geográfica Mitchell \& Lambert (2015) han notado que la falta de una base de conocimiento en el modelo del profesor técnico es lo que se encuentra restringiendo el profesionalismo de los profesores. Reduciendo los referentes que ellos tendrían para navegar sus ambientes de trabajo, lo que por consiguiente reforzaría la noción individualizada del "buen profesor" en la práctica docente. Respecto a esto Young \& Muller (2014) proponen que los profesionales imbuidos en el modelo técnico son valorados por lo que "pueden hacer", más que por lo que "saben hacer". Como Brooks (2016: 101) señala, "la realidad laboral de los profesores es que las influencias desde la cultura escolar o educacional pueden ejercer de seguido una influencia en el aula, limitando las oportunidades de los profesores para mantener este balance". Esto indica que las actuales presiones al trabajo docente enfatizan un entendimiento individual de la geografía que puede ser perjudicial para el desarrollo del campo de la educación geográfica.

Aunque la profesión docente consiste eminentemente en el ejercicio individual en el aula, Clandinin \& Connelly (1995) han enfatizado la existencia de una dimensión social de la práctica docente. Su analogía respecto a los "paisajes profesionales" que cambian en la medida que un profesor se mueve dentro y fuera del aula da cabida a un entendimiento donde la profesionalidad docente se sitúa en espacios múltiples y simultáneos. Brooks $(2007 ; 2016)$ utiliza este argumento como base para precisar sobre las múltiples relaciones entre una dimensión micro de la práctica educativa y otra meso y macro que forma parte un complejo sistema de culturas. "Fuera de su asignatura y disciplina académica lo profesores pueden ser influenciados por tres contextos culturales: el horizonte educacional, su contexto escolar y personal" (Brooks, 2016: 30). Esta idea de "culturas de influencia" sintetiza una noción inherente dentro de la investigación en educación geográfica, donde se considera que cada práctica es informada por diferentes cuerpos de conocimiento (Salinas_Silva et al., 2016). Toda práctica docente, sin importar cuan neutral o tacita sea declarada, expresa acomodación y adaptación en la interacción con diferentes actores y sus códigos sociales (Bernstein, 2000). En efecto, "un profesor puede ser parte de una cultura escolar o disciplinar que activamente está en desacuerdo con las ideas dominantes de una cultura educacional general, y aun así, participar de ambas" (Brooks 2016: 29). Esto no representa contradicción, sino la presencia de contextos profesionales en disputa que están asociados permanentemente a los distintos espacios de la docencia, y que no se limitan solamente a las coyunturas de un cambio curricular o ajustes en la estructura organizacional de las escuelas (Alexandre, 2009). 
En esta sección se ha considerado las discusiones provenientes de los campos de la didáctica y curriculum making para demostrar que la práctica docente para la educación geográfica no es un evento aislado y es mucho más compleja que los eventos que ocurren en el aula. Tanto la práctica docente, como la práctica profesional docente apelan a fenómenos similares referidos al aprendizaje de los estudiantes, pero requieren ser vistos de forma separada debido a que los profesores despliegan estrategias y roles distintos en ambos ámbitos.

\section{Discusión: Hacia una teorización de la "experticia" en educación geográfica}

La preocupación sobre la utilización del término "experticia" viene de la necesidad de comprender la práctica de aula conectada con su contexto profesional. El cual se encuentra dominado por una lógica de rendición de cuentas que ha creado las condiciones para que se cuestione la pertinencia de la enseñanza a través de asignaturas $y$, de paso, la utilidad de la geografía escolar (Alexandre, 2016; Mitchell \& Lambert, 2015; Salinas-Silva et al., 2016; Young \& Muller, 2014). Tal escenario ha promovido una discusión sobre la capacidad de los profesores para agenciarse con las problemáticas educativas de su comunidad tomando como punto de apoyo su "experticia" en un campo específico del conocimiento (Arenas \& Salinas Silva, 2013; Roberts, 2014; Solem et al., 2013; Castellar, 2011; Young \& Lambert, 2015). Esto sugiere la capacidad del término para conectar ideas provenientes de los espacios profesionales de los profesores con los de otros campos de conocimiento. Pero también habla de su utilidad como vehículo para comunicar las ideas desarrolladas en la investigación en educación geográfica hacia otros campos de la educación y las didácticas específicas.

El término "Experticia" es ampliamente utilizado pero pocas veces definido en educación geográfica. Aparece en una gama de investigaciones en curriculum making y didáctica (Arenas et al., 2016; Brooks, 2010; Downs, 1994; Rawling, 2004) encontrándose comúnmente asociado al conocimiento de los profesores sobre la materia, lo que se suele utilizar como factor de diferenciación entre profesores especialistas y no-especialistas (generalistas) en una disciplina (Downs, 1994). No obstante, hay indicios provenientes de la investigación en educación primaria de la geografía y la discusión que se ha generado en torno al pensamiento geográfico que apuntan a la necesidad de una comprensión diferente del término.

Tanto las discusiones sobre la práctica docente como de su profesionalidad se han referido a la "experticia" de los profesores como un ámbito más complejo que el de la práctica, asociándola con el conocimiento disciplinar pero también con la destrezas adquiridas a partir de la experiencia. En este sentido, Collins \& Evans (2002) proponen que la mirada sobre la "experticia" se debe centrar en el proceso de especialización sobre un campo determinado que resulta en un set de conocimientos o habilidades. Como estos elementos varían contextualmente, tanto Edwards (2010) como Young \& Muller (2014) enfatizan la dimensión procesual de la especialización más que en el set fijo de conocimientos que pueden ser genéricos o inaplicables en algunos contextos.

La investigación en educación primaria de la geografía ha permitido ampliar la noción de especialización con la que se considera a los profesores. La educación primaria o básica de la 
geografía en Iberoamérica (Souto \& Navarro, 2016) comprende en la mayoría de los casos una educación inicial de los profesores como generalistas, lo que puede afectar la profundidad de la preparación en conocimientos específicos de las disciplinas informando la docencia. Especialmente en los casos donde la geografía comparte el espacio curricular con otras disciplinas (Salinas-Silva et al., 2015). En diferentes contextos se ha evidenciado que esto afecta la identidad profesional de los docentes (Brooks, 2016; Castellar, 2011) y su auto-eficacia enseñando geografía (Harte \& Reitano, 2015). No obstante, la condición de "especialista" se presenta de forma ambigua, ya que no se distingue en qué medida la especialización es parte de la calificación como producto de un programa de formación o como parte del proceso de especialización en la práctica. Tal distinción es resaltada por Martin (2008a: 14) en Inglaterra, quien establece que "mientras la mayoría de los aspirantes a profesores de primaria no son geógrafos, incluso aquellos que tienen un grado en geografía parecen tener dificultades utilizando sus concepciones de geografía en la práctica". La "experticia" de estos profesores parece venir de la comprensión sobre las geografías de sus estudiantes "de las experiencias en su ambiente, su conciencia espacial y, en menor medida, su comprensión del mundo" (Catling \& Willy, 2009: 177). De acuerdo a Martin (2008b) estas reflexiones provienen en parte de las necesidades de aprendizaje de los estudiantes en un determinado grupo de edad, influyendo en la posición que los profesores de primaria toman respecto de la geografía.

El tipo de conocimiento sobre el cual los profesores desarrollan su "experticia" afecta el estatus que gozan en su contexto escolar. Por ejemplo, contextos escolares con teorías piagetianas imperantes, pueden comprender el aprendizaje de los estudiantes como una serie de etapas de desarrollo cognitivo que sugieren un proceso progresivo donde las fases iniciales son básicas en su complejidad (Roberts, 2003). En este marco, una enseñanza que se basa en la geografía de los niños puede ser atribuida con un menor estatus debido al uso de un tipo de conocimiento circunstancial o experiencial que es básico, pero que se encuentra en mayor cercanía con el contexto del estudiante (Salinas-Silva et al., 2015). Martin (2008b) plantea que cuando la problemática del estatus emerge, puede estar basada en prejuicio o desconocimiento y no en criterios profesionales. Para contrarrestar esto, se plantea que la enseñanza de la geografía cuenta con mecanismos internos para mantener la calidad de la práctica docente dentro de los parámetros de la disciplina. Los profesores en el aula "se encuentran equipados para ser conscientes, y criticar, sus marcos de referencia tácitos, siendo una parte critica del proceso de desarrollo profesional" (Martin, 2008b: 14). Estatus y conocimiento son elementos que emergen relacionados en la enseñanza de la geografía y en cierta medida influenciando la especialización que los profesores realizarían en sus contextos.

La discusión sobre pensamiento geográfico ha permitido identificar características sobre la "experticia" que son propias de los profesores de geografía. En Estados Unidos, Sudamérica e Inglaterra se ha señalado diversos elementos que provienen de la particularidad de cada contexto, pero que en suma proveen referencias específicas sobre las capacidades asociadas a la geografía en educación.

En Estados Unidos se ha entendido la "experticia" de los profesores como un proceso de crecimiento profesional. Estudios en pensamiento espacial asocian el aprendizaje que los estudiantes deberían desarrollar con la "experticia" que los profesores requerirían para conducirlos en el proceso. En este sentido, para que un individuo se encuentre "alfabetizado 
espacialmente" se debe tomar en cuenta "los niveles de 'experticia' a ser adquiridos e identificar los puntos de referencia para lograr estar alfabetizado. Los cuales pueden ser afirmaciones sobre los conocimientos, habilidades y disposiciones que caracterizan la progresión desde inicial (principiante) a alguien experimentado espacialmente (experto)" (Bednarz \& Kemp, 2011: 21). Esta comprensión sobre la "experticia" de los profesores de geografía ha sido influenciada por los estudios cognitivos sobre práctica docente que definen "experticia" como un atributo de los expertos (Amirault \& Branson, 2012; Kalyuga et al., 2003). Se considera que tiene las siguientes características:

“(1) Las mediciones de capacidades básicas no son consideradas como predictores validos de un desempeño experto en un dominio, (2) el desempeño superior de los expertos se relaciona con un dominio específico, y la transferencia fuera de esa área de experticia es sorprendentemente limitada, y (3) diferencias sistemáticas entre expertos e individuos menos competentes casi siempre reflejan atributos adquiridos por los expertos durante todo el periodo de entrenamiento" (Ericsson et al., 2012: 10).

El énfasis en la condición de experto ha permitido a esta línea de estudios modelar un determinado tipo de desempeño, facilitando su transferencia y la posibilidad de que profesores principiantes repliquen la formar de realizar una práctica docente en su proceso de crecimiento profesional (Wilson et al., 1987). Para Bednarz \& Lee (2011) la modelización sobre el pensamiento espacial está en gran medida relacionada con la compleción de una competencia geoespacial asociada a la literatura en tecnologías geoespaciales. No obstante, la comparación entre competencia y "experticia" puede inducir a error (Vincent, 2008), ya que la primera considera la habilidad para realizar una tarea (Blay, 2013; OECD, 2005; Pages, 2007) y la segunda se centra en el proceso de especialización en un campo (Collins \& Evans, 2007), el cual no es modelable, ya que la experiencia en una comunidad de práctica (Willy, 2017) es -directa o indirectamente- necesaria para definir una particular "experticia".

Una forma diferente de abordar el pensamiento espacial proviene de Sudamérica, donde se le considera socialmente construido en diferentes contextos en vez de ser exclusivamente cognitivo y centrado en el individuo. Debido a esto, podemos encontrar trabajos que se centran en la importancia que tendría para la "experticia" de los profesores alcanzar un dominio sobre el contexto de los estudiantes (Araya Palacios et al., 2015) y que este tendría un fuerte carácter experiencial (Cavalcanti, 1998). En este sentido, la geografía provee una comprensión sobre la construcción de los lugares (Vanzella Castellar, 2011) que permitiría -dentro de los parámetros de la disciplina y la asignatura escolar- poder re-contextualizar lo saberes geográficos (Stefenon, 2017).

Es por esto, que el argumento de Miranda (2016) resulta ser de utilidad, ya que considera la existencia de dos formas de raciocinio espacial que permitirian dar comprensión al fenómeno de re-contextualización. Por un lado, el pensamiento geográfico y por el otro, el pensamiento espacial. El primero especialmente regimentado para su uso y transmisión en la academia y los espacios que la convocan. El segundo, de base experiencial relacionado con el conocimiento práctico e intuitivo con que las personas navegan el mundo, pero albergando estructuras de raciocinio que pueden ser transmitidas como es el caso de algunos oficios como los conductores de medios de transporte. En esta consideración de la doble-dimensión del pensamiento espacial 
Brooks (2013: 79) señala que "presentar estos dos en oposición puede ser poco preciso, ya que hay muchas formas para que se informen y trabajen mutuamente". No obstante, ese elemento de interacción e influencia mutua no es desconocida al reconocerse que existen obstáculos epistemológicos que pueden afectar la comunicación de diferentes cuerpos de conocimiento (Miranda, 2006). En efecto, Garrido (2009: 127) ha argumentado que el espacio educacional tiene un carácter interaccional que actúa como línea comunicante "entre los procesos de enseñanza-aprendizaje y los constructos espaciales que las asignaturas generan". Por ejemplo, el conocimiento indígena (Garrido, 2013) que puede estar presente en algunas comunidades escolares del norte de Chile puede ser considerado epistemológicamente valido (Dentice \& Garrido, 2013) dado que tiene formas de organización propia y medios de comunicación que pueden ser desplegados para desarrollar una comprensión geográfica sobre un problema local (Garrido, 2005). Estos argumentos indican una dimensión social a la "experticia" de los profesores, compuesta por múltiples comprensiones sobre el contexto y basada en la experiencia, pero al mismo tiempo sugiere que experiencia no es equivalente a "experticia", debido a las reorganizaciones que los mismos sujetos y profesores tienen que realizar en la práctica, para hacer posible su comprensión y comunicación.

Se ha argumentado que la "experticia" es altamente dependiente del contexto (Brooks, 2007; Collins \& Evans, 2007) y que la relación de los profesores con el contexto se presenta como una inquietud básica en la enseñanza de la geografía. Como Brooks (2013: 80) plantea:

“En la consideración sobre el 'problema' de definir la geografía para las escuelas, diversos trabajos (...) subrayaron la importancia del contexto. Su traducción desde la geografía académica en una geografía escolar viable es un acto consciente que debería tomar en cuenta las preferencias y significados locales. Esa observación, sostengo, es de vital importancia para la cultura de la educación geográfica, ya que enfatiza el rol de los educadores de geografía para comprender las influencias y preferencias prevalecientes en contextos determinados, y para tomar en cuenta la influencia de la educación geográfica".

Por ello, y como último punto, la discusión sobre el pensamiento geográfico en Inglaterra ha compartido la preocupación que los investigadores sudamericanos tienen por el contexto, identificando la importancia que tiene para los profesores de geografía trabajar con el medio social o ambiental. No obstante, también se plantea el riesgo de sobrepoblar estos tópicos con experiencias personales que no responden ante críticas o escrutinio (Martin, 2008a). Por ejemplo, conceptos como "lugar" (Massey, 1994) son frágiles al ser tratados pedagógicamente, porque hacen referencia a aspectos emotivos relacionados con el entendimiento local que pueden tener los estudiantes et al., 2017). Esto ha sido abordado en la discusión sobre el conocimiento poderoso (powerful knowledge) (Young, 2008, 2011), donde se reconoció la importancia de contar con una estructura de enseñanza a través de asignaturas. Para ello se enfatizó el papel de las disciplinas que se desarrollan en las escuelas como un punto de acceso a conocimiento que no es posible de ser encontrado comúnmente en la vida diaria u otros espacios de la sociedad (Maude, 2016).

La discusión sobre la extensión del pensamiento geográfico como conocimiento poderoso, toma un cariz dicotómico cuando se introduce la necesidad de reconocer que en el desarrollo de comprensión en geografía también se requiere prestar atención al conocimiento cotidiano. 
Para Roberts (2014) se debe reconocer la relación entre aprendiz y ambiente de aprendizaje, así como la posibilidad de que la construcción de conocimiento geográfico pueda ser realizado por los estudiantes fuera del canon convencional de la academia. Algo que Catling \& Martin (2011) llaman etno-conocimiento. Esto ha sido criticado ya que limita la comprensión de los estudiantes para visualizar una geografía más allá de lo local (Young \& Lambert, 2015). No obstante, para Catling (2013: 442) reconocer que los niños tienen conocimiento geográfico permite al profesor darse cuenta de lo que los estudiantes pueden traer al aula de geografía, por ejemplo "que los profesores supiera menos sobre el área de la escuela que sus estudiantes, les ayudó a apreciar que habían otras experticias disponibles en la sala de clases". Es por ello, que tales argumentos encuentran un punto medio en el reconocimiento que la geografía puede proveer herramientas básicas a los profesores no solo para traducir conocimiento científico a los estudiantes, sino que para reconocer también las contribuciones de actores fuera de la academia. En este sentido, la educación geográfica ha hecho propio este argumento en distintos momentos. En Chile Aida Chaparro (1967) resaltó la capacidad de la disciplina para generar comprensión sobre el mundo más allá de lo local y Bill Marsden en Inglaterra (1976) consideró la crítica a nociones parroquiales en el conocimiento como un elemento distintivo de los argumentos geográficos. Lo anterior indica la capacidad de la geografía para ofrecer criterios orientadores a los profesores para desarrollar e identificar "experticia" en sus contextos.

\section{Conclusión}

Se ha visto que el concepto de práctica docente es controversial y un espacio en disputa. La literatura en la escuela-efectiva ha podido instalarse en las concepciones sobre lo que debe ser un "buen profesor" influyendo la generalidad de los ámbitos educacionales. No obstante, se ha constatado que los estudios de curriculum making y didáctica de la geografía proveen referentes para el trabajo de aula del profesorado. En estos estudios se llama la atención sobre teorías que con el fin honesto de mejorar la educación y la experiencia de los estudiantes en la escuela, están al mismo tiempo estrechando la capacidad de los profesores para poder actuar en los espacios educativos. Se ha dado cuenta que en la investigación internacional en el campo de la educación geográfica hay una preocupación común por estrechar los lazos entre la disciplina y las experiencias de aprendizaje de los estudiantes porque la geografía misma, como campo de estudios, dispone de eso como un objeto de estudio. Esto involucra considerar términos como el de "experticia", que sitúa la capacidad de agencia del docente en su rol como especialista articulador de conocimientos y los espacios que les dan significado. Una preocupación eminentemente geográfica, pero con implicancias para todo el sistema escolar.

\section{Referencias bibliográficas}

AINSCOW, M. Towards self-improving school systems: lessons from a city challenge. London: Routledge, 2015.

ALEXANDRE, F. Epistemological awareness and geographical education in Portugal: the practice of newly qualified teachers. International Research in Geographical and Environmental Education, 2009, Vol. 18, No 4, p. 253-259. 
ALEXANDRE, F. The standardization of geography teachers' practices: a journey to self-sustainability and professional identity development. International Research in Geographical and Environmental Education, 2016, Vol. 25, № 2, p. 166-188.

AMIRAULT, R. \& BRANSON, R. Educators and Expertise: A Brief History of Theories and Models. In: ERICSSON, K.; CHARNESS, N.; FELTOVICH, P. \& HOFFMAN, R. (editores). The Cambridge Handbook of Expertise and Expert Performance. Cambridge: Cambridge University Press, 2012, p. 69-86.

ARAYA, $F$. The didactics of geography in the contexts of the decade of sustainable education (2005-2014). Revista de Geografia Norte Grande, 2005, No 34, p. 83-98.

ARAYA, F. Enseñanza, investigación y conocimiento geográfico en contextos escolares formales y no formales. Biblio 3W. Revista Bibliográfica de Geografía y Ciencias Sociales, 2009, Vol. XIV, No 831. Disponible en internet: http://www.ub.edu/geocrit/b3w-831.htm

ARAYA, F.; SOUTO, X. \& HERRERA, Y. The geographical space, a school construction. A case study: the pupils of the Limari Valley (Chile). Scripta Nova-Revista Electronica de Geografia y Ciencias Sociales, 2015, Vol. 19, № 503, p. 1-34.

ARAYA-RAMIREZ, I.; QUIROS-ARIAS, L. \& RUIZ-HERNANDEZ, A. Globalization of Higher Education: Experience of the School of Geographic Sciences, National University, Costa Rica. Revista Geográfica de America Central, 2015, No 55, p. 15-47.

ARENAS, A.; FERNÁNDEZ, H. \& PÉREZ, P. Una educación geográfica para Chile. Santiago de Chile: Sociedad Chilena de Ciencias Geográficas, 2016.

ARENAS, A. \& SALINAS SILVA, V. Giros en la Educación Geográfica: renovación de lo geográfico y lo educativo. Revista de Geografía Norte Grande, 2013, № 56, p. 143-162.

ARENAS, A. Las estrategias, actividades y tareas de enseñanza en el aprendizaje comprensivo de la historia y las ciencias sociales escolares. Casos chilenos. Alcalá de Henares: Universidad de Alcalá, Tesis doctoral, 2009.

ARENAS, A.; SALINAS-SILVA, V.; MARGALEF-GARCÍA, L. \& OTERO-AURISTONDO, M. Fragility of Pedagogical Content Knowledge in Geography. Journal of Geography, 2016, p. 1-10.

AVALOS, B. \& ASSAEL, J. Moving from resistance to agreement: The case of the Chilean teacher performance evaluation. International Journal of Educational Research, 2006, Vol. 45, № 4-5, p. 254-266.

BALL, S.; MAGUIRE, M.; BRAUN, A. \& PERRYMAN, J. How Schools Do Policy. New York: Routledge, 2012.

BARBER, M. \& MOURSHED, M. How the world's best-performing schools come out on top. McKinsey \& Company, 2007. Disponible en Internet:

https://www.mckinsey.com/industries/social-sector/our-insights/how-the-worlds-best-performing-school-systems-come-out-on-top 
BEDNARZ, R.S. \& LEE, J. The components of spatial thinking: empirical evidence. Procedia - Social and Behavioral Sciences, 2011, No 21, p. 103-107.

BEDNARZ, S.W. \& KEMP, K. Understanding and nurturing spatial literacy. Procedia - Social and Behavioral Sciences, 2011, No 21, p. 18-23.

BEDNARZ, S.W.; HEFFRON, S. \& HUYNH, N. A road map for 21st century geography education: Geography education research. Washington: Association of American Geographers, 2013.

BERNSTEIN, B. Pedagogy, Simbolic Control, and Identity. London: Rowman \& Littlefield, 2000.

BLAY, M. Desarrollo de competencias básicas y enseñanza de la geografía en la ESO. Íber. Didáctica de Las Ciencias Sociales, Geografía E Historia, 2013, No 74, p. 17-28.

BROOKS, C. Making the geography curriculum: Reflections on the IGU-CGE London Symposium. International Research in Geographical and Environmental Education, 2013, Vol. 22, No 1, p. 71-88

BROOKS, C. \& HOPWOOD, N. Exploring Issues of Validity in a Study of Geography Teachers' Subject Knowledge. Research in Geographic Education, 2005, No 8, p. 59-72.

BROOKS, C. Towards understanding the influence of subject knowledge in the practice of "expert" geography teachers. London: Institute of Education, University of London, Award of Doctor of Philosophy, 2007.

BROOKS, C. Making the geography curriculum: Reflections on the IGU-CGE London Symposium. International Research in Geographical and Environmental Education, 2013, Vol. 22, № 1, p. 71-88.

BROOKS, C. \& HOPWOOD, N. Exploring Issues of Validity in a Study of Geography Teachers' Subject Knowledge. Research in Geographic Education, 2005, No 8, p. 59-72.

BROOKS, C. Why geography teachers' subject expertise matters. Geography, 2010, Vol. 95, No 3, p. 143-148.

BROOKS, C. Teacher Subject Identity in Professional Practice. London: Routledge, 2016.

CALLAI, H.C. O ensino e a pesquisa da Geografia para os anos iniciais do Ensino Fundamental. Revista Brasileira de Educação em Geografia, 2016, Vol. 6, No 11, p. 6-20.

CATLING, S. Giving younger children voice in primary geography: Empowering pedagogy - A personal perspective. International Research in Geographical and Environmental Education, 2014, Vol. $23, N^{\circ} 4$, p. 350-372.

CATLING, S. Teachers' perspectives on curriculum making in Primary Geography in England. The Curriculum Journal, 2013, Vol. 24, No 3, p. 427-453. 
CATLING, S. \& MARTIN, F. Contesting powerful knowledge: the primary geography curriculum as an articulation between academic and children's (ethno-) geographies. The Curriculum Journal, 2011, Vol. 22, No 3, p. 317-335.

CATLING, S. \& WILLY, T. Teaching Primary Geography. Glasgow: Learning Matters, 2009.

CAVALCANTI, L. Geografia, escola e construção de conhecimentos. Campinas-SP: Papirus, 1998.

CHAPARRO, A. Algunas consideraciones sobre la Enseñanza de la Geografía. Revista Geográfica de Valparaíso, 1967, № 1-5, p. 99-104.

CISTERNA, F. Las ciencias sociales en la educación secundaria chilena: entre la permanencia y el cambio. Íber. Didáctica de las Ciencias Sociales, Geografía e Historia, 1999, № 22, p. 31-40.

CLANDININ, D. \& CONNELLY, M. Teachers' professional knowledge landscapes. New York: Teachers college Press, 1995.

COFFIELD, F. Why the McKinsey reports will not improve school systems. Journal of Education Policy, 2012, Vol. 27, № 1, p. 131-149.

COLL, C. El constructivismo en el aula. Barcelona: Graó, 1993.

COLL, C.; POZO, J.I.; SARABIA, B. \& VALLS, E. Los contenidos en la Reforma (Aula XXI). Spain: Santillana, 1992.

COLLINS, H. \& EVANS, R. The Third Wave of Science Studies: Studies of Expertise and Experience. Social Studies of Science, 2002, Vol. 32, No 2, p. 235-296.

COLLINS, H. \& EVANS, R. Rethinking Expertise. Chicago: The University of Chicago, 2007.

CONWAY-GOMEZ, K. \& ARAYA PALACIOS, F. Discussing the Geography of Sustainable Development through an International Online Collaboration with Students in Chile and the USA. Journal of Geography in Higher Education, 2011, Vol. 35, № 2, p. 265-279.

DANAHER, P.A.; DAVIES, A.; GEORGE-WALKER, L.D.; JONES, J.K.; MATTHEWS, K.J.; MIDGLEY, W. \& BAGULEY, M. Disrupting Disempowerment: Agency in Education. In: Contemporary Capacity-Building in Educational Contexts. London: Palgrave Macmillan, 2014, p. 50-61.

DARLING, L. Quality teaching: what is it and how can it be measured? 2011. Disponible en Internet: https://edpolicy.stanford.edu/sites/default/files/events/materials/ldhscopeteacher-effectiveness. pdf, 20/05/2017

DENTICE, A., \& GARRIDO, M. Espacios educativos: medios de vinculación y dominio. Revista Geográfica de Valparaíso, 2013, № 47, p. 53-66. 
DOWNS, R. Being and Becoming a Geographer - an Agenda for Geography Education. Annals of the Association of American Geographers, 1994, Vol. 84, No 2, p. 175-191.

EDWARDS, A. Being an Expert Professional Practitioner. The Relational Turn in Expertise. London: Springer, 2010.

EGGEN, P. \& KAUCHAK, D. Estrategias docentes. Enseñanza de contenidos curriculares y desarrollo de habilidades de pensamiento. México: Fondo de Cultura Económica, 2005.

ERICSSON, K.; CHARNESS, N.; FELTOVICH, P. \& HOFFMAN, R. (editors). The Cambridge Handbook of Expertise and Expert Performance. Cambridge: Cambridge University Press, 2012.

ERRÁZURIZ, A.; GONZÁLEZ, J. \& CERECEDA, P. Geografía y Educación Medioambiental. Revista Geografía Norte Grande, 1997, No 24, p. 191-193.

ESTEVES, M. H. Geography Education and Citizenship Education in Portugal. SAGE Open, 2012, Vol. 2, No 4. https://doi.org/10.1177/2158244012471956

GALLASTEGUI, J. Paisaje, observación directa e itinerarios urbanos. Revista de Geografía Espacios, 2015, Vol. 5, No 9, p. 32-46.

GARRIDO, M. The same space for learning and teaching: the urgencies of geography education. Cadernos CEDES, 2005, Vol. 25, No 66, p.137-163.

GARRIDO, M. La espesura del lugar: reflexiones sobre el espacio en el mundo escolar. Santiago de Chile: Ediciones Universidad Academia de Humanismo Cristiano, 2009.

GARRIDO, M. The Place Where Waters Murmur: Taught and Learned Andean Space. Review of International Geographical Education Online, 2013, Vol. 3, No 1, p. 26-55.

GEOGRAPHICAL ASSOCIATION. A different view. Sheffield: GA, 2009.

GLEADLE, P.; CORNELIUS, N. \& PEZET, E. Enterprising Selves: How Governmentality Meets Agency. Organization, 2008, Vol. 15, No 3, p. 307-313.

GONZÁLEZ, I. El conocimiento geográfico e histórico educativo: la construcción de un saber científico. En: ANADÓN BENEDICTO, J.; GONZÁLEZ GALLEGO, I: y MARTíNEZ GARCÍA, P (coordinadora). La geografía y la historia, elementos del medio. Madrid: Ministerio de Educación y Ciencia, 2002, p. 9-100.

HART, C.S.; BIGGERI, M. \& BABIC, B. (editores). Agency and Participation in Childhood and Youth: International Applications of the Capability Approach in Schools and Beyond. London: Bloomsbury Academic, 2014. 
HARTE, W. \& REITANO, P. Pre-service geography teachers' confidence in geographical subject matter knowledge and teaching geographical skills. International Research in Geographical and Environmental Education, 2015, Vol. 24, № 3, p. 223-236.

HUBER, J.; MURPHY, M. \& CLANDININ, D. Places of curriculum making: Narrative inquiries into children's lives in motion. Bingley: Emerald, 2011.

KALYUGA, S.; AYRES, P.; CHANDLER, P. \& SWELLER, J. The expertise reversal effect. Educational Psychologist, 2003, Vol. 38, No 1, p. 23-31.

KLOCKER, N. An example of "thin" agency. Child domestic workers in Tanzania. IN: PANELLI, R.; PUNCH, S. \& ROBSON, E. (editors). Global Perspectives on Rural Childhood and Youth. New York/ London: Taylor \& Francis, 2007, p. 83-94.

LAMBERT, D. Geography in education. Lost in the post? London: Institute of Education, 2009.

LAMBERT, D. \& MORGAN, J. Teaching Geography 11-18: a conceptual approach. London: Open University Press, 2010.

LLANCAVIL, D., \& GONZÁLEZ, J. A pedagogical approach to the teaching of the geographic space. Revista Electrónica Dialogos Educativos, 2014, Vol. 14, № 28, p. 64-93.

MARSDEN, B. Evaluating the geography curriculum. Edinburgh: Oliver \& Boyd, 1976.

MARSDEN, B. On taking the geography out of geographical education: some historical pointers. Geography, 1997, Vol. 82, No 3, p. 241-252.

MARTIN, F. Ethnogeography: towards liberatory geography education. Childrens Geographies, 2008a, Vol. 6, No 4, p. 437-450.

MARTIN, F. Knowledge Bases for Effective Teaching: Beginning Teachers' Development As Teachers of Primary Geography. International Research in Geographical and Environmental Education, 2008b, Vol. 17, No 1, p. 13-39.

MASSEY, D. A Global Sense of Place. Minneapolis: University of Minnesota Press, 1994.

MAUDE, A. What might powerful geographical knowledge look like? Geography, 2016, № 101, p. 70-76.

MCNAMARA, O.; JONES, M. \& MURRAY, J. Framing workplace learning. In: MCNAMARA, O.; JONES, M. \& MURRAY, J. (editors). Workplace Learning in Teacher Education. London: Springer, 2014, p. $1-27$.

MIRANDA, P. Modelo de progresión del aprendizaje geográfico como proceso situado: aulas interculturales como caso de estudio. Santiago de Chile: Facultad de Educación, Pontificia Universidad Católica de Chile, Tesis Doctoral, 2016. 
MIRANDA, P. Paradigmas dominantes en el proceso de enseñanza aprendizaje de la geografía: obstáculos epistemológicos para la enseñanza de la ciencia en el siglo XXI. Presentado en Grupo de Reflexión e Investigación en Didáctica de las Ciencias (GRECIA), Santiago de Chile, 2006.

MITCHELL, D. Geography teachers and curriculum making in "changing times". International Research in Geographical and Environmental Education, 2016, Vol. 25, № 2, p. 121-133.

MITCHELL, D. \& LAMBERT, D. Subject knowledge and teacher preparation in English secondary schools: the case of geography. Teacher Development, 2015, Vol. 19, № 3, p. 365-380.

MONEREO, C. Las estrategias de aprendizaje. Barcelona: Edebé, 1997.

MORENO LACHE, N.; RODRÍGUEZ, A.C. \& PIZZINATO, L. R. Pensar e indagar la construcción social del espacio: balances y retos. Revista Folios, 2013, No 38, p. 141-156.

MORGAN, J. What makes a "good" geography teacher? In: BROOKS, C. (editor). Studying PGCE Geography at M Level: Reflection, Research and Writing for Professional Development. London: Routledge, 2009, p. 23-35.

ORGANISATION FOR ECONOMIC CO-OPERATION AND DEVELOPMENT (OECD). The definition and selection of key competencies. Paris: OECD, 2005.

ORGANISATION FOR ECONOMIC CO-OPERATION AND DEVELOPMENT (OECD). Teaching and Learning International Survey TALIS 2013 Conceptual framework. Paris: OECD, 2013.

PAGES, J. Un itinerario por el mundo de las competencias en ciencias sociales, geografía e historia a través de distintos currículos. Íber. Didáctica de las Ciencias Sociales, Geografía e Historia, 2007, No 52 , p. 29-39.

PAULSEN, A.; INFANTE, M.; SALAZAR, A. \& VIZCARRA, R. Narrando la vulnerabilidad escolar: performatividad, espacio y territorio. Literatura y Lingüística, 2012, No 27, p. 281-308.

POZO, J.I. \& POSTIGO, Y. Los procedimientos como contenidos escolares. Barcelona: Edebé, 2000.

PUTTICK, S. An analysis of individual and departmental geographical stories, and their role in sustaining teachers. International Research in Geographical and Environmental Education, 2016, Vol. $25, N^{\circ} 2$, p. 134-150.

RAWLING, E. Changing the subject. The impact of national policy on school geography 1980-2000. Sheffield: Geographical Association, 2004.

REYES J.L.; CAMPOS M.J.; OSANDÓN M.L. \& MUÑOZ L.C. El profesorado y su rol en la formación de los nuevos ciudadanos: desfases entre las comprensiones, las actuaciones y las expectativas. Estudios Pedagógicos (Valdivia), 2013, Vol. 39, № 1, p. 217-237.

ROBERTS, M. Interpretations of the geography national curriculum: A common curriculum for all? Journal of Curriculum Studies, 1995, Vol. 27, No 2, p. 187-205. 
ROBERTS, M. Learning through enquiry: Making sense of geography in the key stage 3 classroom. Sheffield: Geographical Association, 2003.

ROBERTS, M. Geography through Enquiry: An approach to teaching and learning in the secondary school. Sheffield: Geographical Association, 2013.

ROBERTS, M. Powerful knowledge and geographical education. The Curriculum Journal, 2014, Vol. 25, No 2, p. 187-209.

SABANI, C. \& MOREIRA, A. Educación para la Sustentabilidad: las Reservas de la Biosfera como espacios de reconexión con la Vida. En: BORSDORF, A. \& MOREIRA, A. (editores). Reservas de la Biosfera de Chile - Laboratorios para la Sustentabilidad. Santiago de Chile: Instituto de Geografía, Pontificia Universidad Católica de Chile - Instituto Interdisciplinario de Investigación sobre la montaña, Academia de Ciencias Austríaca, 2014, p. 296-313.

SALGADO, V. Ensino de Geografia. Espacios. Revista de Geografía, 2012, Vol. 2, No 3, p. 111-113.

SALINAS-SILVA, V.; ARENAS-MARTIJA, A. \& MARGALEF-GARCIA, L. Non-certified environment and geography subject expertise in Chilean rural educators. International Research in Geographical and Environmental Education, 2016, Vol. 25, No 2, p. 105-120.

SALINAS-SILVA, V.; ARENAS-MARTIJA, A. \& RAMIREZ, L. Getting Back to Basics: Is the Knowledge of School Geography Powerful in Chile? In: BROOKS, C.; BUTT, G. \& FARGHER, M. (editors). The power of geographical thinking. London: Springer, 2017, p. 181-198.

SALINAS-SILVA, V.; PEREZ-GALLARDO, P. \& ARENAS-MARTIJA, A. Defining Primary Geography from Teachers' Expertise: What Chilean Teachers Mean by Geography. Review of International Geographical Education Online, 2015, Vol. 5, № 2, p. 166-184.

SANTOS, M. The return of the territory. In Milton Santos: A pioneer in critical geography from the global south. Dordrecht: Springer, 2017.

SCOFFHAM, S. (editor). Primary geography handbook (Rev. and updated ed.). Sheffield: Geographical Association, 2010.

SEOW, T. Reconciling discourse about geography and teaching geography: the case of Singapore pre-service teachers. International Research in Geographical and Environmental Education, 2016, Vol. 25, No 2, p. 151-165.

SOLEM, M., LAMBERT, D., \& TANI, S. Geocapabilities: Toward An International Framework for Researching the Purposes and Values of Geography Education. Review of International Geographical Education Online, 2013, 3(3), 214-229.

SOUTO, X.M. Didáctica de la Geografía y Currículo Escolar. En: GONZÁLEZ, R.; TORRES, M. \& GAI$T E, M$. (editors). Innovación en la enseñanza de la geografía ante los desafíos sociales y territoriales. Zaragoza: Institución Fernando El Católico, 2013, p. 121-147. 
SOUTO, X.M. \& NAVARRO, Y. La eclosión de la enseñanza de geografía en Brasil: una guía para no perderse. Revista Brasileira de Educação em Geografia, 2016, Vol. 6, № 12, p. 8-35.

SOUZA CAVALCANTI, L. The school geography in Brazil and challenges for the teaching practice. Geoenseñanza, 2012, Vol. 17, No 1, p. 23-38.

STEFENON, D. Desigualdades educacionais e esvaziamento curricular: um estudo a partir do caso da recontextualização dos saberes geográficos na escola. São Paulo: Programa de Pós-Graduação em Educação, Faculdade de Educação da Universidade de São Paulo, Tese Doutorado, 2017.

STENGEL, B. Academic discipline' and "school subject": contestable curricular concepts. Journal of Curricular Studies, 1997, Vol. 29, No 5, p. 568-602.

VANZELLA CASTELLAR, S. A superação dos limites para uma educação geográfica significativa: um estudo sobre a e na cidade. Revista Geográfica de América Central, 2011, No 2, p. 1-25.

VASQUEZ LEYTON, G. The curricular bases 2013: current challenges for initial training and historical learning of history teachers in Chile. Educar em Revista, 2016, No 60, p. 147-160.

VERGARA, C., \& COFRÉ, H. Pedagogical Content Knowledge: the missing paradigm in pre-service and in-service science teachers in Chile? Estudios Pedagógicos, 2014, Vol. XL, No 1, p. 323-338.

VINCENT, L. Differentiating Competence, Capability and Capacity. Innovating Perspectives, 2008, Vol. 16, No 3, p. 1-2.

WILLY, T. Meeting the needs of School Direct? Is the School Direct Tuition Fee route enriching the experience of student teachers and providing a high quality home-grown workforce? A case study exploring student and newly qualified teachers experiences of participating in the School Direct Tuition Fee route with a Teaching School Alliance. London: Institute of Education, University College London, Doctorate in Education, 2017.

WILSON, S. M., SHULMAN, L. S., \& RICHERT, A. E. "150 different ways" of knowing: Representations of knowledge in teaching. In: Calderhead, J. (editor). Exploring reachers' thinking. London: CasseII, 1987, p. 104-124.

YOUNG, M. Bringing knowledge back in: From social constructivism to social realism in the sociology of education. Oxon: Routledge, 2008.

YOUNG, M. What are schools for? Educaçâo Sociedade \& Culturas, 2011, № 32, p. 145-155.

YOUNG, M. \& LAMBERT, D. Knowledge and the Future School. Curriculum and Social Justice. London: Bloomsbury, 2015.

YOUNG, M. \& MILLER, J. (editors). Knowledge, Expertise and the Professions. London: Routledge, 2014. 